\title{
Long-term high light stress induces leaf senescence in wheat (Triticum aestivum L.)
}

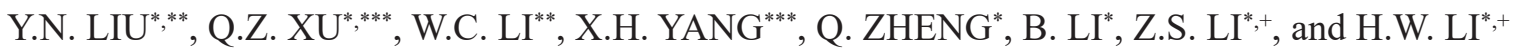 \\ State Key Laboratory of Plant Cell and Chromosome Engineering, Institute of Genetics and Developmental Biology, \\ Innovative Academy of Seed Design, Chinese Academy of Sciences, 100101 Beijing, China* \\ College of Life Sciences, Henan Normal University, Xinxiang 453007, Henan, China** \\ Key Laboratory of Crop Biology, Shandong Agricultural University, 271018 Taian, China***
}

\begin{abstract}
In order to explore the association of long-term high light (HL) with leaf senescence in wheat, two wheat genotypes Xiaoyan 54 (HL-tolerant) and Jing 411 (HL-sensitive) grown in low light were subjected to HL for 2-8 d. The results showed that 6-8 d of HL led to dramatic decline of chlorophyll content, photochemical efficiency, and activity of Rubisco and antioxidant enzymes. Meanwhile, the content of malondialdehyde (MDA), soluble proteins, soluble sugars, superoxide anion, and ascorbic acid increased markedly. Additionally, the expression of senescence-associated genes (SAGs) was induced by HL. Xiaoyan 54 accumulated less MDA, soluble sugars, and superoxide anions but showed a higher ratio of reduced to oxidized ascorbic acid than that in Jing 411 under long-term HL stress. Moreover, most of the assayed SAGs were less induced in Xiaoyan 54 than that in Jing 411. Altogether, the reduction of antioxidant enzyme activities and accumulation of soluble sugars may result in long-term HL-induced leaf senescence.
\end{abstract}

Additional key words: carotenoids; chlorophyll fluorescence; photooxidative stress; reactive oxygen species; Triticum aestivum L.

\section{Introduction}

Wheat is a staple food source that contributes one fifth of calories and proteins of consumption for the world population (Braun et al. 2010). However, in the last decade wheat yield increased below an annual rate of $1 \%$ which does not match the predicated increase at the annual rate of $1.7 \%$ until 2050 for the increasing population (Rosegrant and Agcaoili 2010, Zheng et al. 2011). To meet the large gap, radiation-use efficiency (RUE) is considered an important approach for wheat genetic yield improvement (Horton 2000, Richards 2000, Foulkes et al. 2011, Parry et al. 2011, Reynolds et al. 2012). Photooxidative stress induced by HL, a determining factor for RUE improvement, is a significant constraint for realization of wheat yield potential as it downregulates $\mathrm{CO}_{2}$ assimilation rate and promotes leaf senescence (De La Mata et al. 2013, Juvany et al. 2013, Pintó-Marijuan and MunnéBosch 2014). Therefore, it enables wheat genetic yield improvement via RUE improvement to understand the physiological and genetic mechanisms of tolerance to HLinduced photooxidative stress in wheat.

HL more than utilized for photosynthesis promotes the generation of reactive oxygen species (ROS), which have deleterious effects on photosynthetic apparatus and ultimately reduce growth and photoproductivity (Krause 1988, Long et al. 1994). When plants are transferred from low light (LL) to HL, a lot of changes occur in photosynthetic apparatus involved in short-term and long-term responses (Bailey et al. 2001, Murchie et al. 2002, 2005). Short-term responses take place on a timescale of seconds to minutes and include the state transition (Allen 1995), regulation of PSII photochemical efficiency (Horton et al. 1996), and activation of Rubisco (Salvucci and Ogren 1996). Long-term responses involve the turnover of chloroplast proteins to modulate composition and function of the photosynthetic apparatus (Murchie et al. 2002, 2005). The photosynthetic acclimation of Arabidopsis to

$\overline{\text { Received }} 23$ August 2018, accepted 21 November 2018.

${ }^{+}$Corresponding author; phone: +86-10-64806607, fax: +86-10-64806605, e-mail: zsli@genetics.ac.cn, hwli@genetics.ac.cn.

Abbreviations: APX - ascorbate peroxidase; AsA - ascorbic acid; Car - carotenoids; CAT - catalase; Chl - chlorophyll; DAP - days after planting; DAT - days after transfer; DHA - oxidized ascorbic acid; $\mathrm{DI}_{0} / \mathrm{CS}$ - dissipated energy flux per cross section; $\mathrm{ET}_{0} / \mathrm{CS}$ - electron transport flux per cross section; FM - fresh mass; $F_{v} / F_{m}$ - maximal quantum yield of PSII photochemistry; HL - high light; J411 Jing 411; LL - low light; MDA - malondialdehyde; NPQ - nonphotochemical quenching; PEPC - phosphoenolpyruvate carboxylase; PI - performance index; POD - peroxidase; $\mathrm{qPCR}$ - quantitative polymerase chain reaction; $\mathrm{RC} / \mathrm{CS}_{\mathrm{m}}$ - density of reaction center per excited cross section; ROS - reactive oxygen species; RUE - radiation-use efficiency; SAG - senescence-associated gene; SOD superoxide dismutase; SS - soluble sugars; TCA - trichloroacetic acid; $\mathrm{TR}_{\mathrm{o}} / \mathrm{CS}$ - trapped energy flux per cross section; XY54 Xiaoyan 54.

Acknowledgements: This study was supported by the Natural Science Foundation of China (No. 31571644) and the National Key Basic Research Program (No. 2015CB150106), and the State Key Laboratory of Plant Cell and Chromosome Engineering (PCCEKF-2016-03). 
light intensity performs LL and HL responses (Bailey et al. 2001). Generally, LL responses maximize the efficiency of light capture, while HL responses elevate the lightsaturated rates of photosynthesis and avoid the potential damage of photooxidative stress (Murchie et al. 2005). Photooxidative stress usually results in the decrease of chlorophyll (Chl) content, maximal quantum yield of PSII photochemistry $\left(\mathrm{F}_{\mathrm{v}} / \mathrm{F}_{\mathrm{m}}\right)$, antioxidants, and antioxidant enzymes as well as the enhancement of energy dissipation as heat, ROS, malondialdehyde (MDA), etc., which can be used as markers for leaf senescence (Pintó-Marijuan and Munné-Bosch 2014)

In plants, the susceptibility to photooxidative stress is determined by leaf development (Juvany et al. 2013). For example, the senescing flag leaf of wheat enhances sensitivity to HL and suffers from photooxidative stress during the grain-filling stage (Lu et al. 2001, 2002, 2003; Dai et al. 2004). The downregulation of photosynthetic efficiency of senescing leaves is considered a kind of photoprotection process associated with nonphotochemical quenching (NPQ) via xanthophyll cycle to prevent the damage of photooxidative stress (Lu et al. 2001, 2002, 2003; Dai et al. 2004). However, with the progression of senescence, the decline of NPQ and ROS detoxification of antioxidant enzymes enhances the susceptibility to photooxidative stress and accelerates leaf senescence in turn (Ohe et al. 2005, Juvany et al. 2013). Hence, for wheat plants during the late grain-filling stage, the causal association of photooxidative stress with leaf senescence of flag leaves is complex and difficult to make a clear conclusion due to the accompanying developmental leaf senescence. The tolerance to photooxidative stress induced by $\mathrm{HL}$ is genotype-dependent in cereal plants including rice (Jiao et al. 2001, 2003) and wheat (Wang et al. 2000, Yang et al. 2006, 2007; Li et al. 2010b, Chen et al. 2011). Compared with the HL-tolerant genotypes, the HL-sensitive genotypes are prone to premature leaf senescence (Jiao et al. 2003, Li et al. 2010b). However, the direct evidence of HL-induced senescence lacked up till now. The objectives in the present study were (1) to study the photosynthetic, antioxidant, and expressional responses of senescence-associated genes ( $S A G \mathrm{~s}$ ) and (2) to compare the responses of HL-tolerant and HL-sensitive wheat genotypes to long-term HL stress.

\section{Materials and methods}

Plant material and growing conditions: Two Chinese winter wheat Triticum aestivum L. cv. Xiaoyan 54 (XY54) and Jing 411 (J411) were used in this study. XY54, developed from a distant hybridization between tall wheatgrass (Thinopyrum ponticum, $2 n=10 x=70$ ) and common wheat ( $T$. aestivum, $2 n=42$ ), maintains relatively high photosynthetic efficiency under stress conditions (Li et al. 2008), while J411, a northern Chinese high-yielding variety, shows the high productivity and photosynthetic efficiency under nonstress conditions. Particularly, XY54 was tolerant, while J411 was sensitive to HL-induced photooxidative stress (Wang et al. 2000, Yang et al. 2006, Chen et al. 2011).
After seeds were sterilized with $1.5 \% \mathrm{H}_{2} \mathrm{O}_{2}$ overnight at room temperature, they were transferred to a nylon net in a plastic plate and grown in a growth chamber (HP1000GS, Wuhan Ruihua Instrument \& Equipment Co., China) for one week. The 7-d seedlings were then transplanted in a plastic box with nutrition medium as described by Li et al. (2017) which was renewed every three days. The growth conditions were set as: $20 / 15^{\circ} \mathrm{C}$ (day/night), 14/10-h light/dark cycle with PPFD of $300 \mu \mathrm{mol} \mathrm{m}{ }^{-2} \mathrm{~s}^{-1}$ and relative humidity of $40-60 \%$.

At $22 \mathrm{~d}$ after planting (DAP), the seedlings were separated into two parts. One was subjected to PPFD of $800 \mu \mathrm{mol} \mathrm{m} \mathrm{m}^{-2} \mathrm{~s}^{-1}$ provided by compact fluorescent light bulbs (F55BX/840, GE Lighting Co., USA) at $18-20^{\circ} \mathrm{C}$ and the relative humidity of $40-60 \%$ in a growth incubator (E36HO, Percival Scientific, USA). The first leaves were sampled for measurements at $0,2,4,6$, and $8 \mathrm{~d}$ after transfer from LL to HL, respectively. Meanwhile, another part of seedlings was still grown under PPFD of $300 \mu \mathrm{mol}$ $\mathrm{m}^{-2} \mathrm{~s}^{-1}$ and the first leaves were used at 30 DAP as LL control (LL8d).

Photosynthetic pigments: The content of Chl $(a+b), \mathrm{Chl} a$, $\mathrm{Chl} b$, and carotenoids (Car) were determined according to Arnon (1949) and Lichtenthaler and Wellburn (1983) with small modifications. About $0.05 \mathrm{~g}$ of leaf sample was extracted in $1.5 \mathrm{ml}$ of $80 \%$ acetone in darkness at room temperature for three days when it fully turned to white. Then, the extracts were spectrophotometrically measured at $470,645,646$, and $663 \mathrm{~nm}$ with a microplate reader (SpectraMax190, Molecular Devices, USA).

Chl a fluorescence was measured with a Handy-PEA fluorometer (Hansatech Instruments Ltd., UK). The middle parts of leaves were measured immediately after dark adaption for $20 \mathrm{~min}$ which is long enough to completely relax all nonphotochemical quenching of fluorescence. The saturated flash light intensity was set at PPFD of 3,000 $\mu \mathrm{mol} \mathrm{m} \mathrm{m}^{-2} \mathrm{~s}^{-1}$ and the flash light duration was $1 \mathrm{~s}$. The fluorescence parameters including $\mathrm{F}_{\mathrm{v}} / \mathrm{F}_{\mathrm{m}}$, performance index on absorption basis $\left(\mathrm{PI}_{\mathrm{ABS}}\right)$, maximum trapped energy exciton per active PSII reaction center $\left(\mathrm{TR}_{\mathrm{o}} / \mathrm{RC}\right)$, electron transport flux from $\mathrm{Q}_{\mathrm{A}^{-}}$to plastoquinone (PQ) per active PSII reaction center $\left(\mathrm{ET}_{\mathrm{o}} / \mathrm{RC}\right)$, dissipated energy flux other than trapping per active PSII reaction center $\left(\mathrm{DI}_{\mathrm{o}} / \mathrm{RC}\right)$, and density of reaction center per excited cross section $\left(\mathrm{RC} / \mathrm{CS}_{\mathrm{m}}\right)$ were obtained with $P E A$ Plus software (v. 1.10) and computed following Strasser et al. $(1995,2004)$.

Activity of Rubisco and PEPC: Rubisco (EC 4.1.1.39) was assayed according to Khairy et al. (2016) with some modifications. About $0.05 \mathrm{~g}$ of leaf sample was homogenized in liquid nitrogen and extracted with $0.5 \mathrm{ml}$ of a buffer consisting of $50 \mathrm{mM}$ BTP (pH 7.2), $10 \mathrm{mM} \mathrm{NaHCO} 3,10 \mathrm{mM} \mathrm{MgCl} 2,1 \mathrm{mM}$ EDTA, 0.5 mMATP, 10 mMDTT, 1 mM PMSF, 1 mMbenzamidine, $0.01 \mathrm{mM}$ leupeptin, $1.5 \%$ PVPP, and $3 \mathrm{mM}$ MBT. Rubisco activity was spectrophotometrically measured with a reaction buffer consisting of $1 \mathrm{M}$ Tris- $\mathrm{HCl}(\mathrm{pH} 7.2)$, 
6 mM NADH, $0.1 \mathrm{M} \mathrm{GSH}, 0.5 \mathrm{M} \mathrm{KHCO}_{3}, 0.5 \%$ glyceraldehyde-3-phosphate dehydrogenase, 10 units per $20 \mu \mathrm{l}$ of 3-phosphoglycerate kinase, $0.05 \% \alpha$-glycerophosphate dehydrogenase-triose phosphate isomerase, $25 \mathrm{mM}$ ribulose 1,5-bisphosphate (RuBP), 0.2 M ATP, 0.5 M MgCl , and $10 \mu \mathrm{l}$ of crude enzyme extract in a total volume of $0.2 \mathrm{ml}$. The decrease of absorbance at $340 \mathrm{~nm}$ in $5 \mathrm{~min}$ was recorded to compute Rubisco activity. One unit of Rubisco activity was defined as $1 \mathrm{nmol}(\mathrm{NADH})$ per $1 \mathrm{~min}$ per $1 \mathrm{mg}$ (protein) at $25^{\circ} \mathrm{C}$.

Phosphoenolpyruvate carboxylase (PEPC, EC 4.1.1.31) activity was determined according to Zhang et al. (2008) with small modifications. PEPC was extracted with $0.5 \mathrm{ml}$ of the extraction medium consisting of $0.1 \mathrm{M}$ Tris- $\mathrm{HCl}$ ( $\mathrm{pH} 7.8$ ), $10 \mathrm{mM} \mathrm{MgCl}_{2}, 1 \mathrm{mM}$ ethylenediaminetetraacetic acid, $20 \mathrm{mM}$ mercaptoethanol, 10\% (w/v) glycerin, and $1 \%$ polyvinylpyrrolidone. Then, $10 \mu \mathrm{l}$ of crude PEPC extract was added to a final volume of $0.2 \mathrm{ml}$ of the reaction buffer containing $50 \mathrm{mM}$ Tris- $\mathrm{HCl}(\mathrm{pH}$ 7.8), $10 \mathrm{mM} \mathrm{MgCl}_{2}, 0.25 \mathrm{mM}$ EDTA, $5.0 \mathrm{mM} \mathrm{NaHCO}_{3}, 2.0 \mathrm{mM}$ dithiothreitol, $4 \mathrm{U} \mathrm{MDH}, 0.1 \mathrm{mM} \mathrm{NADH}$, and $2.0 \mathrm{mM}$ PEP. The decrease of absorbance at $340 \mathrm{~nm}$ in $5 \mathrm{~min}$ was recorded to compute PEPC activity. One unit of PEPC activity was defined as $1 \mathrm{nmol}(\mathrm{NADH})$ per $1 \mathrm{~min}$ per $1 \mathrm{mg}$ (protein) at $25^{\circ} \mathrm{C}$.

MDA and soluble sugar content: About $0.05 \mathrm{~g}$ of leaf samples were homogenized with a TissueLyser II (Qiagen, USA) and extracted with $1 \mathrm{ml}$ of $5 \%$ trichloroacetic acid (TCA). After centrifugation at $12,000 \mathrm{rpm}$ for $15 \mathrm{~min}$, the supernatant was used to measure MDA and soluble sugars (SS) according to Ledwożyw et al. (1986). The supernatant of $200 \mu \mathrm{l}$ was mixed with equal volume of $0.6 \%$ thiobarbituric acid dissolved in $5 \%$ TCA, followed by incubation in boiling water for $15 \mathrm{~min}$. After cooling on ice for $10 \mathrm{~min}$ and centrifugation at $12,000 \mathrm{rpm}$ for $5 \mathrm{~min}, \mathrm{MDA}$ and SS concentration were determined per fresh mass (FM) by recording absorbance at 450,532 , and $600 \mathrm{~nm}$, respectively, according to Zhao et al. (1994).

Activity of antioxidant enzymes and superoxide anion and ascorbic acid contents: After $0.05 \mathrm{~g}$ of leaf sample was grounded in liquid nitrogen, the crude enzyme was extracted with $0.5 \mathrm{ml}$ of $50 \mathrm{mM}$ potassium phosphate buffer $(\mathrm{pH}$ 7.0). Followed by centrifugation at $12,000 \mathrm{rpm}$, the supernatant was used for measurements of the antioxidant enzyme activities. Superoxide dismutase (SOD, EC 1.15.1.1) activity was measured by recording the absorbance at $560 \mathrm{~nm}$ according to García-Triana et al. (2010). Catalase (CAT, EC 1.11.1.6) activity was determined by recording the decrease of absorbance at $240 \mathrm{~nm}$ per min following Sima et al. (2011). Ascorbate peroxidase (APX, EC 1.11.1.11) activity was measured by recording the decrease of absorbance at $290 \mathrm{~nm}$ wavelength per min according to Ullah et al. (2016). Peroxidase (POD, EC 1.11.1.7) activity was measured by recording the absorbance of $470 \mathrm{~nm}$ of wavelength according to Wang et al. (2014). The activity of the antioxidant enzymes were calculated per $\operatorname{mg}$ (protein).

The superoxide anion $\left(\mathrm{O}_{2}{ }^{--}\right)$content was determined by recording the decrease of absorbance at $530 \mathrm{~nm}$ wavelength per min following De la Fuente et al. (1995). The content of reduced ascorbic acid (AsA) and oxidized ascorbic acid (DHA) were determined per FM according to Imai et al. (2008) with small modification.

RNA extraction and first strand cDNA synthesis: Total RNA was isolated with TRIzol Reagent (Life Technologies, USA) and the first strand cDNA was synthesized with SuperScript III reverse transcriptase (Invitrogen, USA). About $2 \mu \mathrm{g}$ of total RNA was denatured at $65^{\circ} \mathrm{C}$ for $5 \mathrm{~min}$ in the presence of $0.5 \mu \mathrm{g}$ of oligo $(\mathrm{dT})_{12-18}$ primers and $1 \mu \mathrm{l}$ of $10 \mathrm{mM}$ dNTPs. Subsequently, the tubes were immediately chilled on ice and reverse transcribed with 200 units of SuperScript III reverse transcriptase, $4 \mu \mathrm{l}$ of first-strand buffer, $1 \mu \mathrm{l}$ of $0.1 \mathrm{M}$ DTT, and $1 \mu \mathrm{l}$ of RNaseOUT recombinant RNase inhibitor in a total volume of $20 \mu \mathrm{l}$ at $50^{\circ} \mathrm{C}$ for $60 \mathrm{~min}$. The cDNA products were diluted to $80 \mu \mathrm{l}$ before use.

Gene expression analysis: The quantitative polymerase chain reaction (qPCR) assays were conducted with the StepOnePlus TM Real-Time PCR Systems (Thermo Fisher Scientific, USA) using PowerUp SYBR Green Master Mix (Thermo Fisher Scientific, USA) in a final volume of $10 \mu \mathrm{l}$ including $2 \mu \mathrm{l}$ of cDNA and appropriate primer pairs. The qPCR primers were designed according to the deposited wheat mRNA sequences using DNAMAN software (v. 5.2.2). The oligonucleotide qPCR primer sequences were listed in Table $1 \mathrm{~S}$ (supplement). The primer sequences for TaSAG1-8 were designed according to Kajimura et al. (2010). TaSAG12, TaSAG13, and TaSAG18 were homologous to AtSAG12 (At5g45890), AtSAG13 (At2G29350), and AtSAG18 (At1G71190), respectively. The qPCR primer sequences for TaActin taken as an internal control reference gene and TaNAM were described by Uauy et al. (2006). The relative expression was following the comparative $C_{\mathrm{T}}$ (the threshold cycle of qPCR) method (Schmittgen and Livak 2008). Four replicates were performed for each sample. The specificity of the unique amplification product was determined by melting curve analysis.

Statistical analysis: At least three independent biological experiments were carried out. Data were represented as mean $\pm \mathrm{SD}$. One-way analysis of variance $(A N O V A)$ and multiple comparisons analysis were conducted by using SPSS statistical software (v. 13.0, IBM). For multiple comparisons the LSD method for equal variance assumed was taken. Figures were created with the software SigmaPlot (v. 10.0, Systat Software Inc.).

\section{Results}

Photosynthetic pigments: The changes in the content of Chl and Car were determined in XY54 and J411 when they were transferred from LL to HL for $2-8 \mathrm{~d}$ (Fig. 1). For both cultivars, the content of Chl $(a+b)$, 


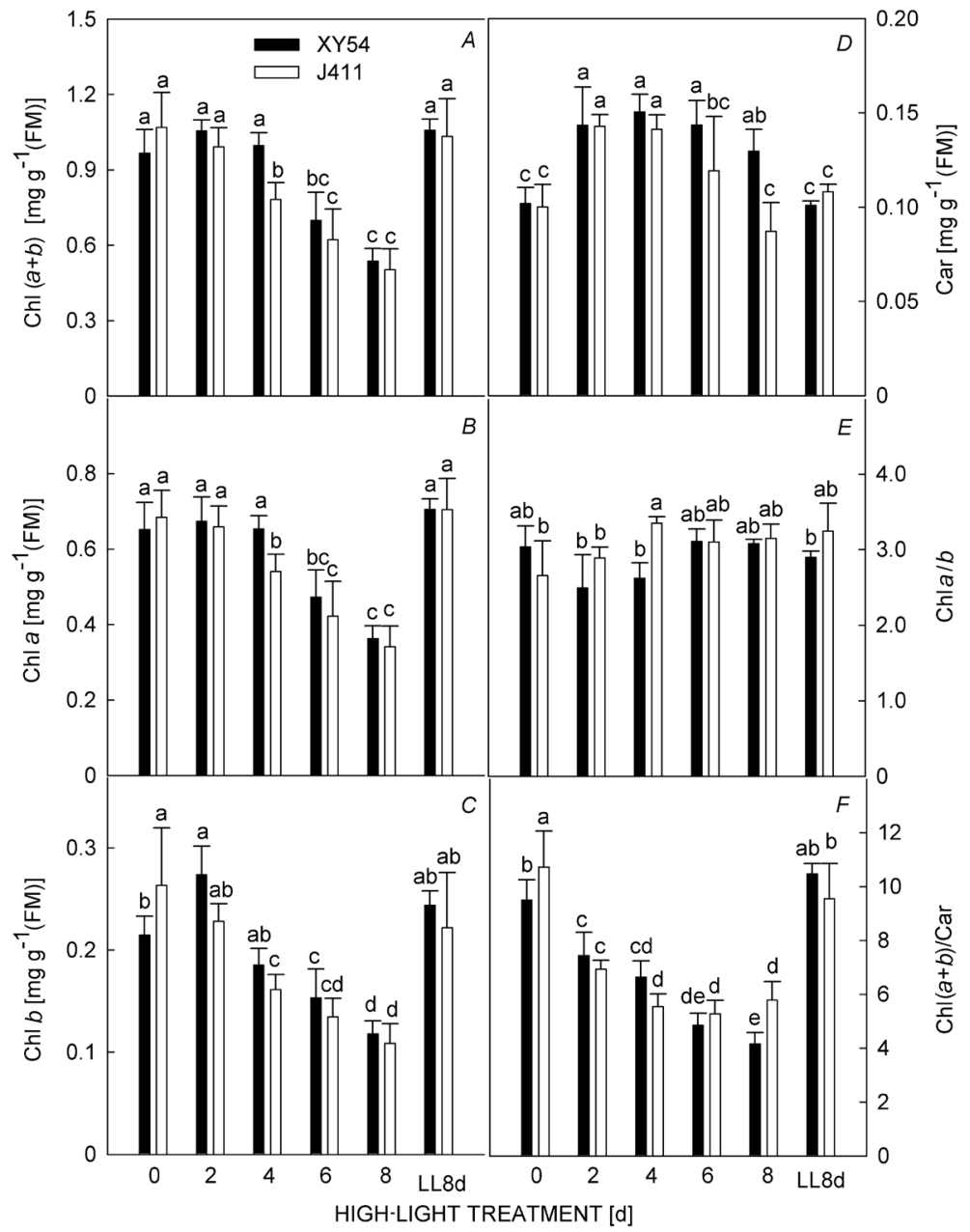

Fig. 1. The changes in content of total chlorophyll (Chl) $(a+b)(A)$, Chl $a(B)$, Chl $b(C)$, carotenoids (Car) $(D)$, Chl $a / b$ ratio $(E)$, and $\mathrm{Chl}(a+b) / \mathrm{Car}$ ratio $(F)$ in two wheat cultivars responding to longterm high light (HL) stress. Data are represented as mean $\pm \mathrm{SD}$, different small letters indicate significant difference at $P<0.05$. XY54 - HL-tolerant cultivar Xiaoyan 54, J411 - HL-sensitive cultivar Jing 411.
Chl $a$, and Chl $b$ declined dramatically from $6 \mathrm{~d}$ after transfer (DAT) with the lowest values being at 8 DAT, while the Car content increased significantly from 2 DAT and then dropped slightly at 8 DAT (Fig. $1 A-D)$. The Chl $a / b$ ratio changed less, while the $\mathrm{Chl}(a+b) / \mathrm{Car}$ ratio declined dramatically from 2 DAT in both cultivars (Fig. 1E,F). The seedlings grown in LL for $8 \mathrm{~d}$, indicated as LL8d, were used as LL control. In comparison with LL8d, 6-8 d of HL treatment accelerated Chl degradation and thus led to leaf senescence. Relatively, the Chl content in J411 started to decrease from 4 DAT, which was $2 \mathrm{~d}$ earlier than that in XY54, demonstrating that J411 was more sensitive to HL stress than XY54.

Chl $\boldsymbol{a}$ fluorescence parameters: The Chl fluorescence parameters including $\mathrm{F}_{\mathrm{v}} / \mathrm{F}_{\mathrm{m}}, \mathrm{PI}_{\mathrm{ABS}}, \mathrm{TR}_{\mathrm{o}} / \mathrm{RC}, \mathrm{ET}_{\mathrm{o}} / \mathrm{RC}, \mathrm{DI}_{\mathrm{o}} /$ $\mathrm{RC}$, and $\mathrm{RC} / \mathrm{CS}_{\mathrm{m}}$ were measured to explore the responses of photochemical activity in wheat to long-term HL stress. The values of $\mathrm{F}_{\mathrm{v}} / \mathrm{F}_{\mathrm{m}}$ (Fig. $2 A$ ), $\mathrm{PI}_{\mathrm{ABS}}$ (Fig. $2 B$ ), $\mathrm{ET}_{\mathrm{o}} /$ $\mathrm{RC}$ (Fig. $2 D$ ), and $\mathrm{RC} / \mathrm{CS}_{\mathrm{m}}$ (Fig. $2 F$ ) in both XY54 and J411 declined extremely when subjected to HL stress for even $2 \mathrm{~d}$, suggesting that at 2 DAT HL stress led to severe repression of PSII photochemical activity and thus occurrence of significant photooxidative stress in both cultivars. With prolonging of HL treatment, the values of
$\mathrm{F}_{\mathrm{v}} / \mathrm{F}_{\mathrm{m}}, \mathrm{PI}_{\mathrm{ABS}}, \mathrm{ET}_{\mathrm{o}} / \mathrm{RC}$, and $\mathrm{RC} / \mathrm{CS}_{\mathrm{m}}$ declined continuously, reflecting the further damage of PSII. In addition, the values of $\mathrm{TR}_{0} / \mathrm{RC}$ in both cultivars decreased at 6-8 DAT (Fig. 2C), indicating that long-term HL stress also repressed trapped energy flux. Meanwhile, the values of $\mathrm{DI}_{\mathrm{o}} / \mathrm{RC}$ increased considerably at 8 DAT (Fig. $2 E$ ), indicating that thermal dissipation was enhanced by long-term HL.

Photosynthetic enzyme activity: The activity of Rubisco and PEPC were assayed to investigate the photosynthetic activity in wheat responding to long-term HL stress. For both XY54 and J411, the content of soluble proteins increased consistently when they were subjected to longterm HL stress (Fig. 3A). The Rubisco activity increased about twofold at 2 DAT and then declined with prolonging of HL treatment, with the lowest value being at 8 DAT for both cultivars. Compared with 0 DAT and LL8d, the Rubisco activity in both cultivars was severely inhibited when subjected to HL for $8 \mathrm{~d}$ (Fig. $3 B$ ), indicating that long-term HL stress largely inhibited photosynthesis. During HL treatment, the PEPC activity in XY54 kept relatively stable and declined slightly at 8 DAT, while in J411, it decreased markedly and consistently from 4 DAT. In addition, it was lower in J411 than that in XY54 at all sampling time points (Fig. $3 \mathrm{C}$ ). 


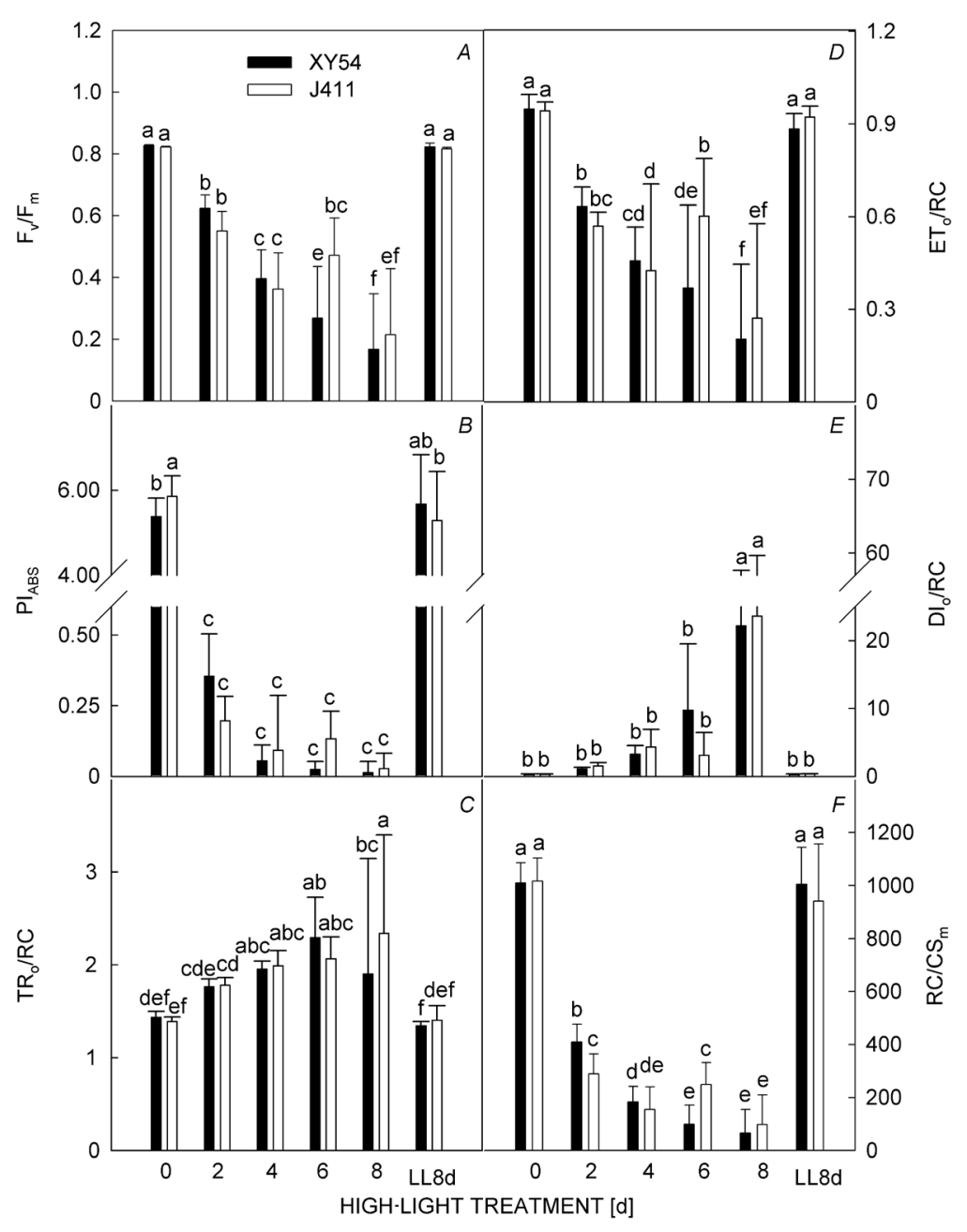

Fig. 2. The changes in maximal quantum yield of PSII photochemistry $\left(\mathrm{F}_{\mathrm{v}} / \mathrm{F}_{\mathrm{m}}\right)$ $(A)$, performance index on absorption basis $\left(\mathrm{PI}_{\mathrm{ABS}}\right)(B)$, maximum trapped energy flux per active PSII reaction center $\left(\mathrm{TR}_{\mathrm{o}} / \mathrm{RC}\right)(C)$, electron transport flux from $\mathrm{Q}_{\mathrm{A}^{-}}$to PQ per active PSII reaction center $\left(\mathrm{ET}_{\mathrm{o}} / \mathrm{RC}\right)(D)$, dissipated energy flux other than trapping per active PSII reaction center $\left(\mathrm{DI}_{0} / \mathrm{RC}\right)(E)$, and density of reaction center per excited cross section $\left(\mathrm{RC} / \mathrm{CS}_{\mathrm{m}}\right)(F)$ in two wheat cultivars responding to long-term high light (HL) stress. Data are represented as mean $\pm \mathrm{SD}$, different small letters indicate significant difference at $P<0.05$. XY5 $4-$ HLtolerant cultivar Xiaoyan 54, J411 - HL-sensitive cultivar Jing 411.

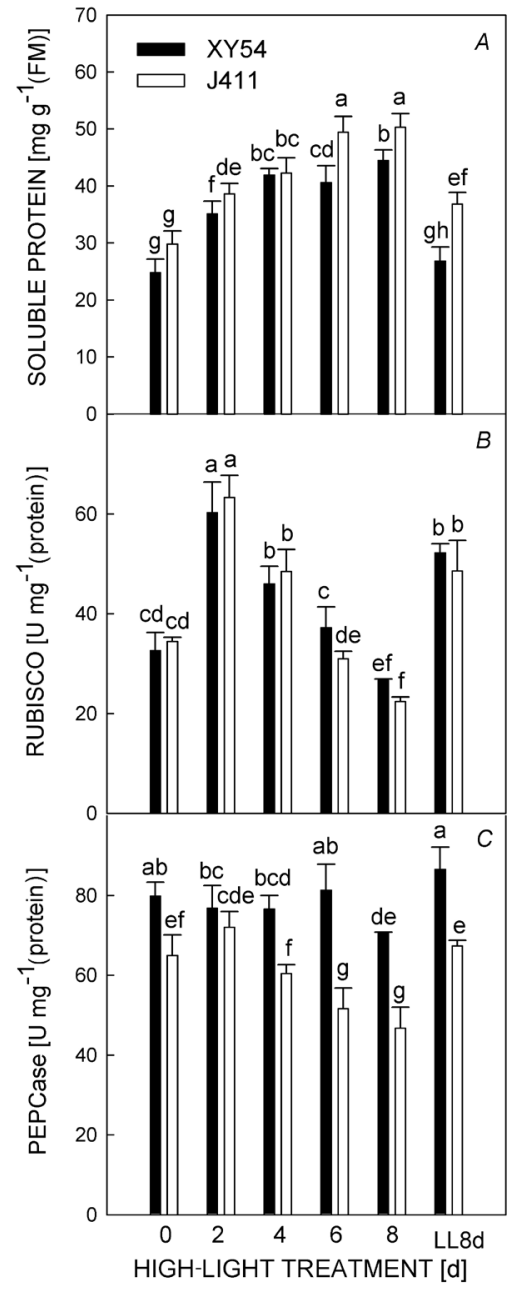

Fig. 3. The changes in soluble protein content $(A)$, activity of Rubisco $(B)$, and activity of phosphoenolpyruvate carboxylase (PEPC) $(C)$ in two wheat cultivars responding to long-term high light (HL) stress. Data are represented as mean $\pm \mathrm{SD}$, different small letters indicate significant difference at $P<0.05$. XY54 - HL-tolerant cultivar Xiaoyan 54, J411 - HL-sensitive cultivar Jing 411.
MDA, superoxide anion, and ascorbic acid contents: In order to explore the oxidative responses of wheat to long-term HL stress, the amount of MDA, SS, $\mathrm{O}_{2}{ }^{--}$, and ascorbic acid in both XY54 and J411 were assayed after HL treatment. As shown in Fig. 4A, the MDA content in both cultivars was enhanced substantially and it was significantly higher in J411 than that in XY54, especially at 6-8 DAT, indicating that J411 may suffer more severe lipid peroxidation than XY54. Meanwhile, HL treatment also resulted in significant accumulation of SS and $\mathrm{O}_{2}{ }^{--}$ in both cultivars (Fig. $4 B, C$ ), indicating that the overproduction of ROS such as $\mathrm{O}_{2}^{--}$may be ascribed to the accumulation of lipid peroxidation product, MDA. Further analysis demonstrated that when XY54 and J411 were exposed to long-term HL stress, the AsA content increased, while DHA content decreased (Fig. 4D,E), and ultimately resulting in the increase of AsA/DHA ratio for both cultivars (Fig. $4 F$ ). The AsA/DHA ratio reflecting reduction/oxidation state was lower in J411 than that in XY54, suggesting that the lower reduction state may result in more accumulation of $\mathrm{O}_{2}{ }^{-}$in $\mathrm{J} 411$.

Activity of antioxidant enzymes: The activity of antioxidant enzymes including SOD, CAT, APX, and POD was assayed to study the responses of antioxidant enzymes in wheat to long-term HL stress. As shown in Fig. $5 A$, the SOD activity in XY54 declined significantly at 4-8 DAT, while it increased at 2 DAT and then decreased to the pretreatment level in J411. In LL, the SOD activity was lower in J411 than that in XY54, while in HL, no significant difference was observed for both cultivars. The CAT activity in J411 decreased significantly at 6-8 DAT, while 


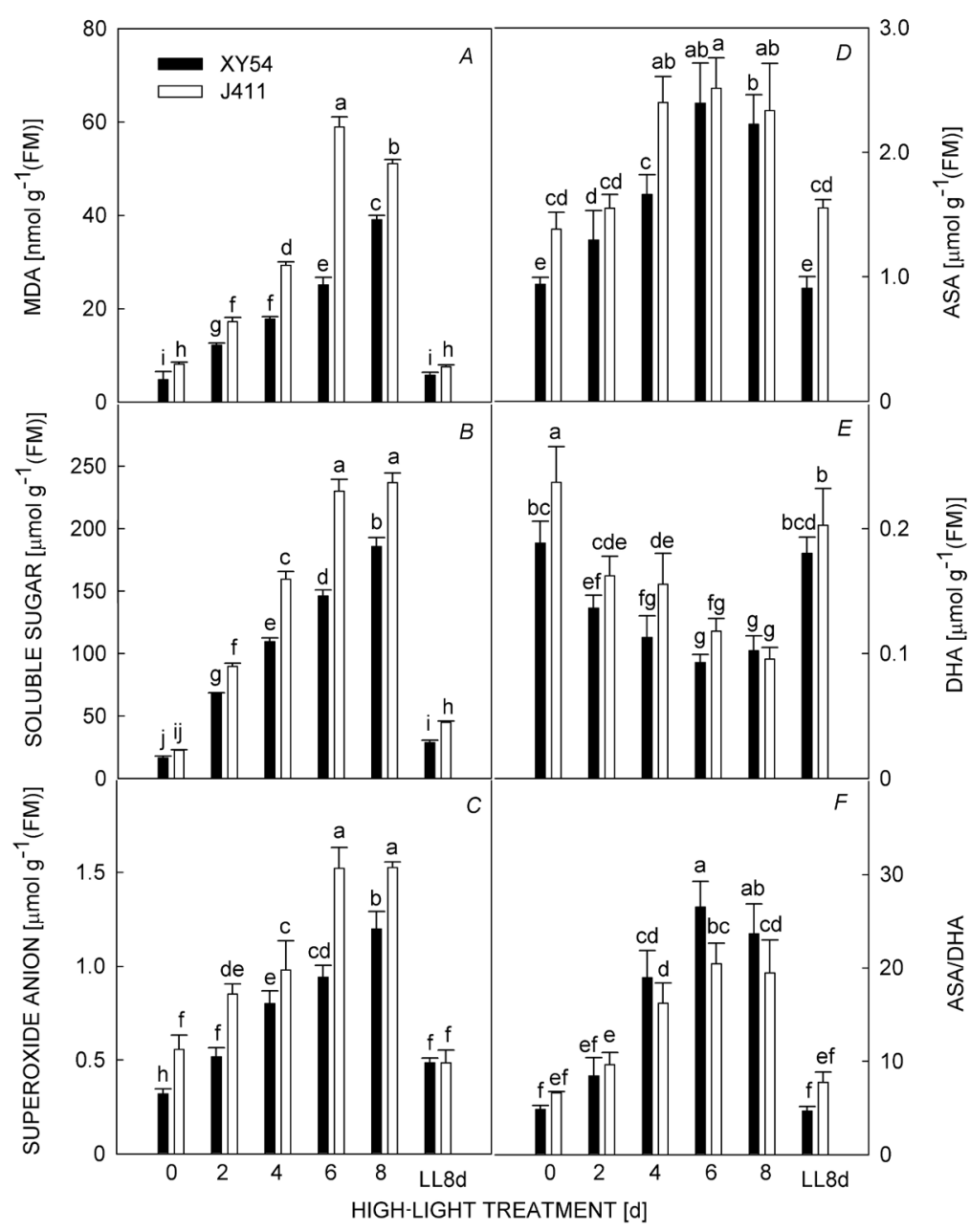

Fig. 4. The changes in the content of malondialdehyde $(A)$, soluble sugar $(B)$, superoxide anion $(C)$, reduced ascorbic acid (AsA) $(D)$, oxidized ascorbic acid (DHA) $(E)$, and ratio of AsA/DHA $(F)$ in two wheat cultivars responding to long-term high light (HL) stress. Data are represented as mean $\pm \mathrm{SD}$, different small letters indicate significant difference at $P<0.05$. XY54 - HL-tolerant cultivar Xiaoyan 54, J411 - HL-sensitive cultivar Jing 411. it decreased markedly from 2 DAT in XY54 (Fig. 5B), indicating that the CAT activity in XY54 was more sensitive to long-term HL stress than that in J411. The APX activity in both cultivars decreased dramatically at 4-8 DAT and it was significantly higher in J411 than that in XY54 at 8 DAT (Fig. 5C). The POD activity in both XY54 and J411 decreased consistently when they were exposed to HL stress and it was significantly higher in XY54 than in J411 at every sampling time points (Fig. 5D). It appeared that the accumulation of ROS such as $\mathrm{O}_{2}{ }^{-}$may be associated with the depression of antioxidant enzymes activity under long-term HL stress.

The expressional responses of $S A G$ s to long-term HL stress: To explore the progression of leaf senescence due to long-term HL stress, the expression profiling of 12 wheat $S A G$ s was assayed in XY54 and J411 when subjected to HL stress. As illustrated in Fig. 6, the expression of TaSAG1 (Fig. 6A) and TaSAG5 (Fig. 6E) was induced and peaked in both cultivars when exposed to HL for $8 \mathrm{~d}$. However, the expression of TaSAG2-4 (Fig. 6B-D), TaSAG7-8 (Fig. 6G,H), TaSAG12-13 (Fig. 6I,J), and TaSAG18 (Fig. $6 K$ ) was induced and peaked at $6 \mathrm{~d}$ of HL treatment. Meanwhile, the expression of TaNAM increased and peaked at 2 and 8 DAT (Fig. $6 L$ ), respectively. Hence, the induction of TaSAGs by HL treatment for 6-8 d reflects the occurrence of leaf senescence induced by long-term HL stress. Furthermore, the induction of 9 of the 12 investigated TaSAGs was more pronounced in J411 than that in XY54 at 6 and/or 8 DAT, suggesting that J411 was more prone to long-term HL-induced senescence than the HL-tolerant cultivar XY54.

\section{Discussion}

Photooxidative stress induced by long-term HL is common for the upmost flag leaves of wheat canopy, especially during the late grain-filling stage, which downregulates $\mathrm{CO}_{2}$ assimilation rate, promotes leaf senescence, and results in grain yield loss ultimately. The situation in the fields is too complex to explore the promotion of long-term HL stress to leaf senescence for developmental and/or environmental cues. In order to investigate the association of long-term HL with leaf senescence, we examined the photosynthetic, antioxidant, and $S A G \mathrm{~s}$ expression responses of wheat seedlings to long-term HL stress under controllable laboratory conditions. Previous study revealed that after HL treatment for less than two days, the content of Chl $(a+b)$ and Chl $a$ remained unchanged, while Chl $b$ significantly decreased, resulting in the increase of Chl $a / b$ (Li et al. 2010a). In this work, when LL-grown wheat seedlings were transferred to HL for $6-8 \mathrm{~d}$, the content of 


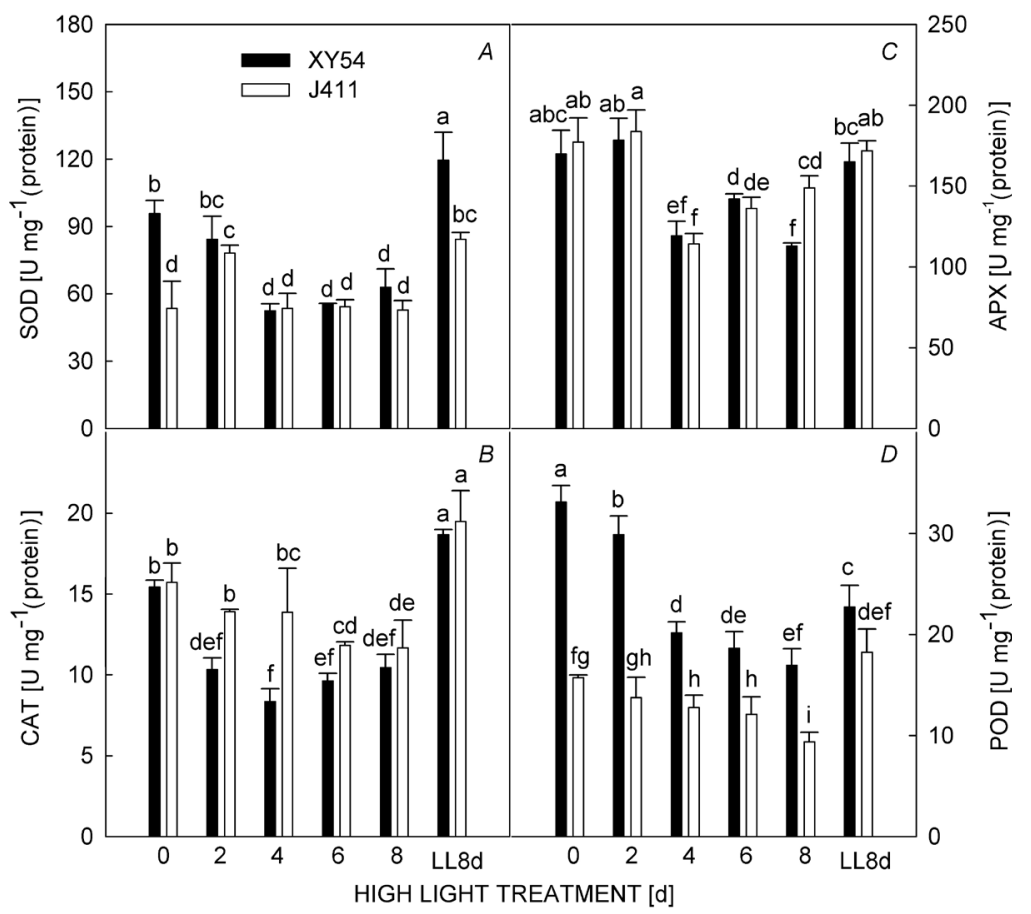

Fig. 5. The changes in the activity of superoxide dismutase (SOD) $(A)$, catalase (CAT) $(B)$, ascorbate peroxidase $(\mathrm{APX})(C)$, and peroxidase $(\mathrm{POD})(D)$ in two wheat cultivars responding to long-term high light (HL) stress. Data are represented as mean $\pm \mathrm{SD}$, different small letters indicate significant difference at $P<0.05$. XY54 - HL-tolerant cultivar Xiaoyan 54, J411 - HL-sensitive cultivar Jing 411.
Chl $(a+b)$, Chl $a$, and Chl $b$ declined markedly, which was indicative of the occurrence of leaf senescence. However, the content of Car increased substantially from 2 DAT and then slightly declined at 6 and 8 DAT. The decrease of the Chl content indicates the reduction of antenna size, while the increase of the Car content indicates its protection role in response to HL stress (Neale and Melis 1986, Biswal 1995). HL treatment for 6-8 d resulted in substantial degradation of $\mathrm{Chl}$ and photobleaching to some extent, which was consistent with previous studies (Pjon 1981, Raval et al. 1982, Maunders and Brown 1983, Biswal and Biswal 1984, Noodén et al. 1996, Velez-Ramirez et al. 2011). However, at 2 DAT, the increase of $\mathrm{Chl} b$ content in XY54 and Car content in both cultivars may partly occur due to the variation of relative water content (RWC) in sampled leaves to some extent.

$\mathrm{HL}$ treatment for longer than $2 \mathrm{~d}$ led to a substantial decline of $\mathrm{F}_{\mathrm{v}} / \mathrm{F}_{\mathrm{m}}, \mathrm{PI}_{\mathrm{ABS}}, \mathrm{ET}_{\mathrm{o}} / \mathrm{RC}$, and $\mathrm{RC} / \mathrm{CS}_{\mathrm{m}}$ but a considerable increase of $\mathrm{DI}_{\mathrm{o}} / \mathrm{RC}$ at $8 \mathrm{DAT}$, indicating that PSII photochemical efficiency, performance index for energy conservation from photons absorbed by PSII antenna to the reduction of $\mathrm{Q}_{\mathrm{B}}$, electron transport flux from $\mathrm{Q}_{\mathrm{A}}{ }^{-}$to PQ, and density of PSII reaction centers declined, while energy flux dissipated in processes other than trapping per active PSII enhanced (Stirbet and Govindjee 2011, Kalaji et al. 2014, Stirbet et al. 2018). The substantial reduction of PSII photochemical efficiency demonstrated severe photooxidative stress took place for even $2 \mathrm{~d}$ of HL treatment. The HL treatment for longer than $2 \mathrm{~d}$ deteriorated the photooxidative status, which may accelerate leaf senescence. Contrastingly, the values of all the investigated fluorescence parameters in the LL8d control were approximately equal to those before transfer to HL, which was consistent with the variation of photosynthetic pigments, suggesting that PSII photochemical efficiency did not altered after LL for $8 \mathrm{~d}$. Therefore, HL treatment for 6-8 d without other stress indeed resulted in very severe photooxidative stress, which was consistent with the degradation of Chl. The xanthophyll cycle-dependent thermal dissipation plays an important role in response of wheat to HL during developmental senescence in the field (Lu et al. 2001, 2002, 2003). However, with the progression of senescence, another mechanism, possibly inactivation of PSII reaction centers, could act to protect severely senescent leaves from photooxidative stress (Dai et al. 2004). Our data also confirmed that the enhancement of thermal dissipation plays a role in the responses to long-term HL although it was not enough to prevent leaf senescence induced by long-term HL stress.

In parallel, the Rubisco activity in both XY54 and J411 decreased significantly at 8 DAT although it was elevated by $2-4 \mathrm{~d}$ of HL treatment, which was consistent with Sedigheh et al. (2011). The depression of Rubisco activity reflects the inhibition of $\mathrm{CO}_{2}$ assimilation rate, which in turn promotes the generation and damage of ROS. Interestingly, the PEPC activity in J411 decreased substantially at 6-8 DAT, while it remained less changed in XY54 at the same time. Furthermore, the PEPC activity in XY54 was higher than that in J411 at all the assayed time points, suggesting that the PEPC activity may play a role in HL tolerance of XY54. Ding et al. (2012) had previously reported that the transgenic rice overexpressing $P E P C$ gene enhanced photoinhibition tolerance under drought stress conditions. Therefore, PEPC activity maybe the target for wheat RUE improvement through enhancement of HL tolerance.

The long-term HL stress induced degradation of photosynthetic pigments and inhibition of photochemical efficiency and $\mathrm{CO}_{2}$ assimilation enzymes activity promotes the overproduction of ROS which ultimately 


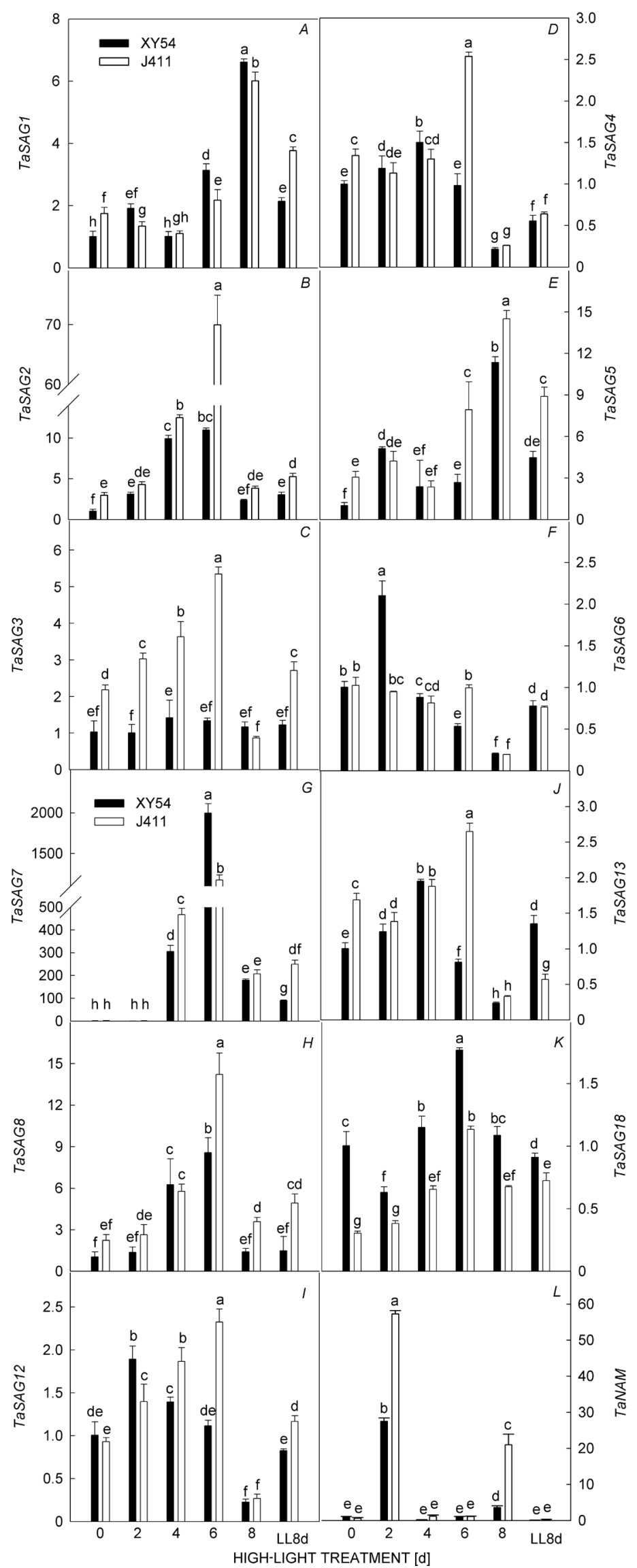

Fig. 6. The expressional responses of senescence associated genes $(S A G \mathrm{~s})$ in two wheat cultivars under long-term high light (HL) stress. Data are represented as mean $\pm \mathrm{SD}$, different small letters indicate significant difference at $P<0.05$. XY54 HL-tolerant cultivar Xiaoyan 54, J411 - HL-sensitive cultivar Jing 411. 
results in the damage of cellular membrane. As shown in Fig. $4 C$, the $\mathrm{O}_{2}^{-}$content in both XY54 and J411 increased with prolonging of $\mathrm{HL}$ treatment. Meantime, the MDA content in both cultivars also increased with $\mathrm{O}_{2}{ }^{-}$ content when they were subjected to long-term HL stress (Fig. 4A). MDA is the products of cellular membrane lipid peroxidation, revealing the damage of ROS to plant cellular membrane system. The overproduction of ROS including $\mathrm{O}_{2}^{--}$and MDA may result from the depression of antioxidant enzymes ( $\mathrm{Ji}$ et al. 2002, Procházková and Wilhelmová 2004, De La Mata et al. 2013). In this work, the activity of antioxidant enzymes including SOD, CAT, APX, and POD in both cultivars were inhibited significantly, although the antioxidants including AsA was increased by long-term HL, which ultimately led to the accumulation of ROS under long-term HL stress. In parallel, when subjected to long-term HL stress, both XY54 and J411 generated a large amount of soluble sugars that is usually considered to induce leaf senescence (Pourtau et al. 2006, Wingler et al. 2006). In addition, the soluble protein content in XY54 and J411 increased significantly after HL treatment for $8 \mathrm{~d}$ which was different from the decline trend in nature senescing flag leaves $(\mathrm{Li}$ et al. 2014). This may be ascribed to the difference between leaf senescence induced by the long-term HL stress and the developmental senescence.

$S A G s$ are usually upregulated during leaf senescence and can be used as marker genes for senescence (Gan and Amasino 1997). For instance, in Arabidopsis, several SAGs including SAG12 and SAG13 have been used as senescence marker genes (Diaz et al. 2005). Uauy et al. (2006) characterized a NAC gene NAM (also termed as $G p c-B 1)$ that regulates leaf senescence and the content of grain protein, zinc, and iron in wheat. Then, Kajimura et al. (2010) isolated nine wheat $S A G$ cDNA clones, named as TaSAG1-9, which are upregulated during natural senescence and dark-induced senescence. These wheat SAGS or homologous SAGS (TaSAG12, TaSAG13, and TaSAG18) were used as senescence markers to study the progression of leaf senescence induced by long-term HL stress (Fig. 6). Most of the assayed wheat SAGs were upregulated by long-term HL stress in comparison with the LL8d control, demonstrating that long-term HL stress indeed induced the leaf senescence. For instance, the expression levels of TaSAG1 encoding an aminotransferase and TaSAG5 encoding a methylcrotonoyl-CoA carboxylase were dramatically induced at 8 DAT, while those of TaSAG2 encoding seed protein B32E, TaSAG7 encoding a putative isocitrate lyase, TaSAG8 encoding a 3-ketoacyl-CoA thiolase-like protein, and TaSAG18 were highly expressed at 6 DAT in both XY54 and J411. However, the expression of TaNAM was induced markedly at 2 and 8 DAT in XY54 and $\mathrm{J} 411$, respectively, which was dramatically induced in senescing flag leaves. The differential expression of wheat $S A G S$ in response to long-term HL may be ascribed to the different roles they played during leaf senescence (Buchanan-Wollaston 1997). In addition, the expression levels and patterns of wheat $S A G S$ differed between XY54 and J411. The mRNA transcripts of nine SAGs (TaSAG2-6, TaSAG8, TaSAG12-13, TaNAM) were highly expressed in
J411 relative to XY54, demonstrating that the HL-sensitive variety J411 was prone to leaf senescence under long-term HL stress.

In conclusion, long-term HL stress resulted in dramatic degradation of chlorophyll, inhibition of PSII photochemical efficiency and Rubisco activity, and overproduction of ROS and MDA due to the repression of antioxidant enzymes activity. In addition, long-term HL stress substantially induced the expression of many $S A G$ s. Therefore, long-term HL stress led to leaf senescence of wheat and the progression of leaf senescence could be postponed in HL-tolerant genotypes.

\section{References}

Allen J.F.: Thylakoid protein phosphorylation, state1-state 2 transitions and photosystem stoichiometry adjustment: Redox control at multiple levels of gene expression. - Physiol. Plantarum 93: 196-205, 1995.

Arnon D.I.: Copper enzymes in isolated chloroplasts. Polyphenoloxidase in Beta vulgaris. - Plant Physiol. 24: 1-15, 1949.

Bailey S., Walters R.G., Jansson S., Horton P.: Acclimation of Arabidopsis thaliana to the light environment: the existence of separate low light and high light responses. - Planta 213: 794-801, 2001.

Biswal B.: Carotenoid catabolism during leaf senescence and its control by light. - J. Photoch. Photobio. B 30: 3-13, 1995.

Biswal U.C., Biswal B.: Photocontrol of leaf senescence. Photochem. Photobiol. 39: 875-879, 1984.

Braun H.J., Atlin G., Payne T.: Multi-location testing as a tool to identify plant response to global climate change. - In: Reynolds M.P. (ed.): Climate Change and Crop Production. Pp. 115-138. CABI Climate Change Series, Surrey 2010.

Buchanan-Wollaston V.: The molecular biology of leaf senescence. - J. Exp. Bot. 48: 181-199, 1997.

Chen X.Y., Li W., Lu Q.T. et al.: The xanthophyll cycle and antioxidative defense system are enhanced in the wheat hybrid subjected to high light stress. - J. Plant Physiol. 168: 1828-1836, 2011.

Dai J., Gao H., Dai Y., Zou Q.: Changes in activity of energy dissipating mechanisms in wheat flag leaves during senescence. - Plant Biol. 6: 171-177, 2004.

De la Fuente M., Hernanz A., Collazos M.E. et al.: Effects of physical exercise and aging on ascorbic acid and superoxide. J. Comp. Physiol. B 165: 315-319, 1995.

De La Mata L., Cabello P., De La Haba P., Agüera E.: Study of the senescence process in primary leaves of sunflower (Helianthus annuus L.) plants under two different light intensities. - Photosynthetica 51: 85-94, 2013.

Diaz C., Purdy S., Christ A. et al.: Characterization of markers to determine the extent and variability of leaf senescence in Arabidopsis. A metabolic profiling approach. - Plant Physiol. 138: 898-908, 2005.

Ding Z.S., Zhou B.Y., Sun X.F., Zhao M.: [High light tolerance is enhanced by overexpressed PEPC in rice under drought stress.] - Acta Agron. Sin. 38: 285-292, 2012. [In Chinese]

Foulkes M.J., Slafer G.A., Davies W.J. et al.: Raising yield potential of wheat. III. Optimizing partitioning to grain while maintaining lodging resistance. - J. Exp. Bot. 62: 469-486, 2011.

Gan S.S., Amasino R.M.: Making sense of senescence. Molecular genetic regulation and manipulation of leaf senescence. Plant Physiol. 113: 313-319, 1997.

García-Triana A., Zenteno-Savín T., Peregrino-Uriarte A.B., 
Yepiz-Plascencia G.: Hypoxia, reoxygenation and cytosolic manganese superoxide dismutase (cMnSOD) silencing in Litopenaeus vannamei: effects on cMnSOD transcripts, superoxide dismutase activity and superoxide anion production capacity. - Dev. Comp. Immunol. 34: 1230-1235, 2010.

Horton P.: Prospects for crop improvement through the genetic manipulation of photosynthesis: morphological and biochemical aspects of light capture. - J. Exp. Bot. 51: 475-485, 2000.

Horton P., Ruban A.V., Walters R.G.: Regulation of light harvesting in green plants. - Annu. Rev. Plant Phys. 47: 655-684, 1996.

Imai, T., Niwa M., Ban Y. et al.: Importance of the L-galactonolactone pool for enhancing the ascorbate content revealed by L-galactonolactone dehydrogenase-overexpressing tobacco plants. - Plant Cell Tiss. Org. 96: 105-112, 2008.

Ji B.H., Zhu S.Q., Jiao D.M.: [Photochemical efficiency of PSII and membrane lipid peroxidation in leaves of indica and japonica rice (Oryza sativa) under chilling temperature and strong light stress conditions.] - Acta Bot. Sin. 44: 139-146, 2002. [In Chinese]

Jiao D.M., Ji B.H., Li X.: Characteristics of chlorophyll fluorescence and membrane-lipid peroxidation during senescence of flag leaf in different cultivars of rice. Photosynthetica 41: 33-41, 2003.

Jiao D.M., Li X.: Cultivar differences in photosynthetic tolerance to photooxidation and shading in rice (Oryza sativa L.). Photosynthetica 39: 167-175, 2001.

Juvany M., Müller M., Munné-Bosch S.: Photo-oxidative stress in emerging and senescing leaves: a mirror image? - J. Exp. Bot. 64: 3087-3098, 2013.

Kajimura T., Mizuno N., Takumi S.: Utility of leaf senescenceassociated gene homologs as developmental markers in common wheat. - Plant Physiol. Bioch. 48: 851-859, 2010.

Kalaji H.M., Schansker G., Ladle R.J. et al.: Frequently asked questions about in vivo chlorophyll fluorescence: practical issues. - Photosynth. Res. 122: 121-158, 2014.

Khairy A.I.H., Oh M.J., Lee S.M. et al.: Nitric oxide overcomes $\mathrm{Cd}$ and $\mathrm{Cu}$ toxicity in in vitro-grown tobacco plants through increasing contents and activities of rubisco and rubisco activase. - Biochimie Open 2: 41-51, 2016.

Krause G.H.: Photoinhibition of photosynthesis. An evaluation of damaging and protective mechanisms. - Physiol. Plantarum 74: 566-574, 1988.

Ledwożyw A., Michalak J., Stepień A., Kadziołka A.: The relationship between plasma triglycerides, cholesterol, total lipids and lipid-peroxidation products during human atherosclerosis. - Clin. Chim. Acta 155: 275-283, 1986.

Li H.W., Li B., Zheng Q., Li Z.S.: [Variation in photosynthetic traits and antioxidant enzyme activities in wheat seedlings transferred from low to high light growth condition.] - Acta Agron. Sin. 36: 449-456, 2010a. [In Chinese]

Li H.W., Tong Y.P., Li B. et al:: Genetic analysis of tolerance to photo-oxidative stress induced by high light in winter wheat (Triticum aestivum L.). - J. Genet. Genomics 37: 399-412, $2010 \mathrm{~b}$.

Li H.W., Wang G., Liu S.D. et al.: Comparative changes in the antioxidant system in the flag leaf of early and normally senescing near-isogenic lines of wheat (Triticum aestivum L.). Plant Cell Rep. 33: 1109-1120, 2014.

Li H.W., Zheng Q., Zhang J. et al.: The analysis of determining factors and evaluation of tolerance to photoinhibition in wheat (Triticum aestivum L.). - Photosynthetica 55: 69-76, 2017.

Li Z.S., Li B., Tong Y.P.: The contribution of distant hybridization with decaploid Agropyron elongatum to wheat improvement in China. - J. Genet. Genomics 35: 451-456, 2008.
Lichtenthaler H.K., Wellburn A.R.: Determinations of total carotenoids and chlorophylls $a$ and $b$ of leaf extracts in different solvents. - Biochem. Soc. T. 11: 591-592, 1983.

Long S.P., Humphries S., Falkowski P.G.: Photoinhibition of photosynthesis in nature. - Annu. Rev. Plant Phys. 45: 633-662, 1994.

Lu C.M., Lu Q.T., Zhang J.H., Kuang T.Y.: Characterization of photosynthetic pigment composition, photosystem II photochemistry and thermal energy dissipation during leaf senescence of wheat plants grown in the field. - J. Exp. Bot. 52: 1805-1810, 2001.

Lu Q.T., Lu C.M., Zhang J.H., Kuang T.Y.: Photosynthesis and chlorophyll $a$ fluorescence during flag leaf senescence of field-grown wheat plants. - J. Plant Physiol. 159: 1173-1178, 2002.

Lu Q.T., Wen X.G., Lu C.M. et al.: Photoinhibition and photoprotection in senescent leaves of field-grown wheat plants. - Plant Physiol. Bioch. 41: 749-754, 2003.

Maunders M.J., Brown S.B.: The effect of light on chlorophyll loss in senescing leaves of sycamore (Acer pseudoplatanus L.). Planta 158: 309-311, 1983.

Murchie E.H., Hubbart S., Chen Y.Z. et al.: Acclimation of rice photosynthesis to irradiance under field conditions. - Plant Physiol. 130: 1999-2010, 2002.

Murchie E.H., Hubbart S., Peng S., Horton P.: Acclimation of photosynthesis to high irradiance in rice: gene expression and interactions with leaf development. - J. Exp. Bot. 56: 449-460, 2005.

Neale P., Melis A.: Algal photosynthetic membrane complexes and the photosynthesis-irradiance curve: a comparison of light-adaptation responses in Chlamydomonas reinhardtii (Chlorophyta). - J. Phycol. 22: 531-538, 1986.

Noodén L.D., Hillsberg J.W., Schneider M.J.: Induction of leaf senescence in Arabidopsis thaliana by long days through a light-dosage effect. - Physiol. Plantarum 96: 491-495, 1996.

Ohe M., Rapolu M., Mieda T. et al.: Decline in leaf photooxidativestress tolerance with age in tobacco.-Plant Sci. 168: 1487-1493, 2005.

Parry M.A.J., Reynolds M., Salvucci M.E. et al.: Raising yield potential of wheat. II. Increasing photosynthetic capacity and efficiency. - J. Exp. Bot. 62: 453-467, 2011.

Pintó-Marijuan M., Munné-Bosch S.: Photo-oxidative stress markers as a measure of abiotic stress-induced leaf senescence: advantages and limitations. - J.Exp. Bot. 65:3845-3857, 2014.

Pjon C.J.: Effects of cyclohexamide and light on leaf senescence in maize and hydrangea. - Plant Cell Physiol. 22: 847-854, 1981.

Pourtau N., Jennings R., Pelzer E. et al.: Effect of sugar-induced senescence on gene expression and implications for the regulation of senescence in Arabidopsis. - Planta 224: 556-568, 2006.

Procházková D., Wilhelmová N.: Changes in antioxidative protection in bean cotyledons during natural and continuous irradiation-acceleratedsenescence.-Biol.Plantarum 48:33-39, 2004.

Raval M.K., Mishra A.N., Biswal U.C.: Changes in the membranes of wheat chloroplasts aging in vitro. - Photobioch. Photobiop. 4: 293-297, 1982.

Reynolds M., Foulkes J., Furbank R. et al.: Achieving yield gains in wheat. - Plant Cell Environ. 35: 1799-1823, 2012.

Richards R.A.: Selectable traits to increase crop photosynthesis and yield of grain crops. - J. Exp. Bot. 51: 447-458, 2000.

Rosegrant M.W., Agcaoili M.: Global Food Demand, Supply, and Price Prospects to 2010. International Food Policy Research Institute, Washington 2010. 
Salvucci M.E., Ogren W.L.: The mechanism of Rubisco activase: Insights from studies of the properties and structure of the enzyme. - Photosynth Res. 47: 1-11, 1996.

Schmittgen T.D., Livak K.J.: Analyzing real-time PCR data by the comparative $C_{\mathrm{T}}$ method. - Nat. Protoc. 3: 1101-1108, 2008.

Sedigheh H.G., Mortazavian M., Norouzian D. et al.: Oxidative stress and leaf senescence. - BMC Res. Notes 4: doi: 10.1186/ 1756-0500-4-477, 2011.

Sima Y.H., Yao J.M., Hou Y.S. et al.: Variations of hydrogen peroxide and catalase expression in Bombyx eggs during diapause initiation and termination.-Arch. Insect. Biochem.77: 72-80, 2011.

Stirbet A., Govindjee: On the relation between the Kautsky effect (chlorophyll $a$ fluorescence induction) and photosystem II: Basis and applications of the OJIP fluorescence transient. J. Photoch. Photobio. B 104: 236-257, 2011.

Stirbet A., Lazar D., Kromdijk J., Govindjee: Chlorophyll a fluorescence induction: Can just a one-second measurement be used to quantify abiotic stress responses? - Photosynthetica 56: 86-104, 2018.

Strasser R.J., Srivastava A., Govindjee: Polyphasic chlorophyll a fluorescence transient in plants and cyanobacteria. Photochem. Photobiol. 61: 32-42, 1995.

Strasser R.J., Tsimilli-Michael M., Srivastava A.: Analysis of chlorophyll $a$ fluorescence transient. - In: Papageorgiou G.C., Govindjee (ed.): Chlorophyll $a$ Fluorescence: A Signature of Photosynthesis. Pp. 321-362. Springer, Dordrecht 2004.

Uauy C., Distelfeld A., Fahima T. et al.: A NAC gene regulating senescence improves grain protein, zinc, and iron content in wheat. - Science 314: 1298-1301, 2006.

Ullah S., Kolo Z., Egbichi I. et al.: Nitric oxide influences glycine betaine content and ascorbate peroxidase activity in maize. S. Afr. J. Bot. 105: 218-225, 2016.
Velez-Ramirez A.I., van Ieperen W., Vreugdenhil D., Millenaar F.F.: Plants under continuous light. - Trends Plant Sci. 16: 310-318, 2011.

Wang L., Wang Y., Wang X. et al.: Regulation of POD activity by pelargonidin during vegetative growth in radish (Raphanus sativus L.). - Sci. Hortic.-Amsterdam 174: 105-111, 2014.

Wang S.W., Xu C.C., Bai K.Z. et al.: [Comparative study on photoinhibition between two wheat genotypes.] - Acta Bot. Sin. 42: 1300-1303, 2000. [In Chinese]

Wingler A., Purdy S., MacLean J.A., Pourtau N.: The role of sugars in integrating environmental signals during the regulation of leaf senescence. - J. Exp. Bot. 57: 391-399, 2006.

Yang X.H., Chen X.Y., Ge Q.Y. et al.: Tolerance of photosynthesis to photoinhibition, high temperature and drought stress in flag leaves of wheat: A comparison between a hybridization line and its parents grown under field conditions. - Plant Sci. 171: 389-397, 2006.

Yang X.H., Chen X.Y., Ge Q.Y. et al.: Characterization of photosynthesis of flag leaves in a wheat hybrid and its parents grown under field conditions. - J. Plant Physiol. 164: 318-326, 2007.

Zhang Y.H., Wang Z.M., Huang Q., Shu W.: Phosphoenolpyruvate carboxylase activity in ear organs is related to protein concentration in grains of winter wheat. - J. Cereal Sci. 47: 386-391, 2008.

Zhao S.J., Xu Z.C., Zou Q. et al.: [Improvement of method for measurement of malondialdehyde in plant tissues.] - Plant Physiol. Commun. 30: 207-210, 1994. [In Chinese]

Zheng T.C., Zhang, X.K., Yin G.H. et al.: Genetic gains in grain yield, net photosynthesis and stomatal conductance achieved in Henan Province of China between 1981 and 2008. - Field Crop. Res. 122: 225-233, 2011.

(C) The authors. This is an open access article distributed under the terms of the Creative Commons BY-NC-ND Licence. 